\title{
PANCREATITE AGUDA GRAVE: RESULTADOS DO TRATAMENTO CIRÚRGICO EM 68 PACIENTES
}

\author{
SEVERE ACUTE PANCREATITIS: RESULTS OF SURGICAL TREATMENT IN 68 \\ PATIENTS
}

\author{
Olívio Louro Costa,TCBC-ES ${ }^{1}$ \\ Maria da Penha Zago-Gomes ${ }^{2}$ \\ Carlos Sandoval Gonçalves ${ }^{2}$
}

\begin{abstract}
RESUMO: Objetivo: Analisar os resultados do tratamento cirúrgico das formas necrosantes, ou graves, da pancreatite aguda e da forma crônica agudizada. Método: Foi preenchido um protocolo, de modo consecutivo e prospectivo, de 68 pacientes operados por pancreatite aguda grave e crônica agudizada, no período de 1982 a 2000. Os pacientes foram classificados em três categorias: 1. Pancreatite aguda biliar; 2 . Pancreatite aguda de causa indeterminada; 3. Pancreatite crônica agudizada. Resultados: As indicações para o tratamento cirúrgico foram: diagnóstico incerto $(32,3 \%)$; necrose infectada $(60,3 \%)$ e para necrosectomia $(7,4 \%)$. As operações realizadas foram: desbridamento de necrose infectada $(70,6 \%)$; operações sobre as vias biliares $(20,6 \%)$ e desbridamento de grandes necroses $(7,4 \%)$. Os óbitos hospitalares incidentes, por categoria foram: 1. Pancreatite aguda biliar $(33,3 \%) ; 2$. Pancreatite aguda indeterminada $(45,0 \%) ; 3$. Pancreatite crônica agudizada $(37,0 \%)$. A média de óbitos foi de $38,2 \%$. Vinte e cinco pacientes foram reoperados, uma ou várias vezes, e nestes a mortalidade foi de 40,0\%. Os abscessos foram responsáveis por 52,0\% dos óbitos e as outras complicações que resultaram em óbitos, sempre evoluiram para infecção. Conclusões: Ocorreu um alto índice de operações por diagnóstico incerto. Esta indicação deve se restringir aos casos onde não seja possível o diagnóstico diferencial com certas causas de abdome agudo. As indicações para tratar precocemente a litíase biliar devem ser evitadas ou reduzidas a situações específicas. As reoperações são freqüentemente indicadas nesses pacientes e a infecção foi a principal causa de morte.
\end{abstract}

Descritores: Pancreatite necrosante aguda; Cirurgia; Avaliação de resultados.

\section{INTRODUÇÃO}

Pancreatite aguda é a inflamação aguda do pâncreas, em resposta a agressões de várias causas, como organismos vivos, processos metabólicos, tóxicos e alterações obstrutivas da papila de Vater ou do próprio ducto pancreático. A doença permite recuperação integral da glândula, tanto do ponto de vista morfológico, quanto funcional, uma vez afastada a causa da afecção. A pancreatite aguda pode ser tão benigna que os sintomas desaparecem antes do diagnóstico ou o paciente pode chegar ao hospi- tal em fase final, e o diagnóstico ser feito na autóp$\operatorname{sia}^{1,2}$. A maioria dos casos se situa entre os dois extremos. Aceitam-se duas formas clínicas bem definidas: forma edematosa, leve ou intersticial e forma necrosante, necro-hemorrágica ou grave. A pancreatite intersticial ocorre em $80 \%$ dos pacientes, com mortalidade de $2 \%$. A forma necrosante ou grave acomete $20 \%$ dos casos e resulta em $40 \%$ de óbitos ${ }^{1}$. A pancreatite aguda grave sempre implica em algum grau de necrose, que pode evoluir para complicações, como abscessos, pseudocistos e falência de órgãos.

1. Professor Adjunto do Departamento de Clínica Cirúrgica/UFES (in memorian).

2. Professores Adjuntos do Departamento de Clínica Médica/UFES.

Recebido em 11/07/2003

Aceito para publicação em 10/02/2004

Trabalho realizado nos Serviços de Clínica Cirúrgica e Gastroenterologia do Hospital Universitário Cassiano Antônio de Moraes (HUCAM), da Universidade Federal do Espírito Santo (UFES). 
A pancreatite crônica está na maioria das vezes ligada ao uso abusivo do etanol e, nestes casos, não há recuperação morfológica e funcional da glândula, mesmo afastada a causa. Admite-se que o surto agudo ocorra no paciente que já tem pancreatite crônica alcoólica e é discutível a ocorrência de crise isolada de pancreatite aguda no paciente que participa de grandes libações ${ }^{3}$. O certo é que o quadro clínico da pancreatite crônica agudizada é semelhante ao das pancreatites de outras etiologias e exige a mesma abordagem terapêutica. O propósito do presente estudo é analisar os resultados do tratamento cirúrgico das formas necrosantes, ou graves da pancreatite aguda e da forma crônica agudizada.

\section{MÉTODO}

Foram analisados 68 pacientes operados por pancreatite aguda e crônica agudizada, no período de 1982 a 2000, através da análise de protocolo individual preenchido prospectivamente. Na fase inicial do estudo o diagnóstico era estabelecido pelos dados clínicos, pelos níveis séricos da amilase, pelas radiografias simples do abdome, pelas radiografias contrastadas do estômago e duodeno, na busca de sinais de compressão de vísceras ôcas. Na medida que se tornaram disponíveis, foram utilizadas a ultrasonografia e a tomografia computadorizada, o que permitiu melhorar a acurácia do diagnóstico. Os pacientes foram classificados em três categorias: 1. Pancreatite aguda biliar (PAB); 2. Pancreatite aguda de causa indeterminada (PAI) e 3. Pancreatite crônica agudizada (PCA). Foi considerado diagnóstico incerto quando a laparotomia foi indicada em vista do diagnóstico sindrômico de abdome agudo, antes que a causa fosse claramente estabelecida. Afora o diagnóstico incerto, não houve indicação de cirurgia de urgência, mesmo na pancreatite aguda grave. Os pacientes foram operados, em geral, no fim da segunda semana, quando apresentavam evidência clínica de infecção. Foram realizadas intervenções sobre as vias biliares nas pancreatites biliares, drenagens externas com drenos de Penrose ou tubulares, às vezes, associadas a tamponamento através de incisões limitadas, suficiente apenas para o desbridamento do foco. Também foram usadas incisões amplas, deixando-se o abdome aberto para desbridamento, e drenagens extraperitoneais, através de incisões transversas nos flancos, sobretudo nas reoperações. As reoperações foram indicadas quando a primeira intervenção não resultou em cura da doença e quando ocorreram outras complicações tratáveis cirurgicamente, como fístulas e obstruções. Os pacientes com pancreatite aguda foram acompanhados enquanto freqüentaram o ambulatório, e aqueles com pancreatite crônica agudizada permaneceram em tratamento no Serviço de Gastroenterologia.

\section{RESULTADOS}

Foram operados 41 pacientes com pancreatite aguda grave e 27 com pancreatite crônica agudizada, o que perfaz o total de 68 casos. A média de idade dos 41 pacientes com pancreatite aguda foi de 46,4 anos, e $26(63,4 \%)$ eram do sexo feminino. A média de idade dos 27 pacientes com pancreatite crônica agudizada foi de 43,7 anos e todos eram do sexo masculino. A indicação clínica para a operação, por categoria de pancreatite aguda encontra-se na Tabela 1. As operações realizadas em 68 pacientes, por categoria de pancreatite aguda, encontram-se na Tabela 2.

Tabela 1 - Indicação clínica da cirurgia por categoria de pancreatite aguda em 68 pacientes.

\begin{tabular}{lllll}
\hline Indicação & PAB & PAI & PCA & Total \\
\hline Diagnóstico incerto & 09 & 10 & 03 & $22(32,3 \%)$ \\
Necrose infectada & 11 & 07 & 23 & $41(60,3 \%)$ \\
Grandes necroses & 01 & 03 & 01 & $05(7,4 \%)$ \\
\hline Total de pacientes & 21 & 20 & 27 & $68(100,0 \%)$ \\
\hline
\end{tabular}

PAB (Pancreatite aguda biliar); PAI (Pancreatite aguda de causa indeterminada);

PCA (Pancreatite crônica agudizada).

Tabela 2 - Operações realizadas em 68 pacientes, por categoria de pancreatite aguda.

\begin{tabular}{lllll}
\hline Operações & PAB & PAI & PCA & Total \\
\hline $\begin{array}{l}\text { Desbridamento de } \\
\text { necrose infectada }\end{array}$ & 06 & 16 & 26 & $48(70,6 \%)$ \\
$\begin{array}{l}\text { Operações sobre } \\
\text { as vias biliares }\end{array}$ & 13 & 01 & 0 & $14(20,6 \%)$ \\
$\begin{array}{l}\text { Desbridamento de } \\
\text { grandes necroses }\end{array}$ & 01 & 03 & 01 & $05(7,4 \%)$ \\
\hline Total & 21 & 20 & 27 & $68(100,0 \%)$ \\
\hline
\end{tabular}


Todas as operações de desbridamento resultaram em drenagem externa. A técnica de abdome aberto, com incisões amplas foi utilizada em 13 pacientes com grandes necroses e infecção muito grave e oito faleceram $(61,3 \%)$. Os óbitos hospitalares por categoria de pancreatite aguda grave, encontram-se na Tabela 3. A mortalidade nos pacientes reoperados foi de 40,0\% (Tabela 4). As causas das reoperações em 25 pacientes encontram-se na Tabela 5.

Tabela 3 - Óbitos hospitalares por categoria de pancreatite aguda.

\begin{tabular}{lll}
\hline Categoria & $\mathbf{N}^{\mathbf{0}}$ pacientes & Óbitos \\
\hline PAB & 21 & $07(33,3 \%)$ \\
PAI & 20 & $09(45,0 \%)$ \\
PCA & 27 & $10(37,0 \%$ \\
\hline Total & 68 & $26(38,2 \%)$ \\
\hline
\end{tabular}

Tabela 4 - Óbitos nos pacientes reoperados por categoria de pancreatite aguda.

\begin{tabular}{lll}
\hline Categoria & Pac. Reoperados & Óbitos \\
\hline PAB & $12(57,1 \%)$ & $03(25,0 \%)$ \\
PAI & $05(25,0 \%)$ & $04(80,0 \%)$ \\
PCA & $08(29,6 \%)$ & $03(37,5 \%)$ \\
\hline Total & $25(36,8 \%)$ & $10(40,0 \%)$ \\
\hline
\end{tabular}

Tabela 5 - Causas das reoperações em 25 pacientes com pancreatite aguda grave.

\begin{tabular}{lcr}
\hline Causas & $\mathbf{N}^{\mathbf{0}}$ reoperações & \% \\
\hline Abscessos & 13 & 52,0 \\
Evisceração & 03 & 12,0 \\
Fístula entérica & 03 & 12,0 \\
Hemorragia cirúrgica & 02 & 8,0 \\
Obstrução intestinal & 02 & 8,0 \\
Outras & 02 & 8,0 \\
\hline Total & 25 & 100,0 \\
\hline
\end{tabular}

\section{DISCUSSÃO}

Foram incluídos os pacientes com pancreatite aguda grave, de etiologia biliar, os de causa indeterminada e aqueles com pancreatite crônica agudizada. Estes últimos apresentavam a mesma necrose e o mesmo quadro clínico das outras categorias.

Conforme se observa na Tabela (1), 32,3\% dos pacientes foram operados com diagnóstico incerto. Isto ocorreu nos doentes internados de urgência, com diagnóstico presuntivo de abdome agudo, por outras causas, ou então pela abordagem emergencial da litíase biliar. Um grupo de 20 pacientes nos quais não se determinou a presença de cálculos, nem tinham história de etilismo, foi considerado como de causa indeterminada. Foram pacientes com a mesma faixa etária daqueles com pancreatite aguda, que apresentavam um quadro clínico mais grave e que foram operados com mais urgência, por diagnóstico incerto. Muitos destes pacientes faleceram no pós-operatório ou tiveram alta e não compareceram ao ambulatório para as revisões e portanto não se conseguiu esclarecer a causa da doença. Supõe-se que eram pacientes com litíase biliar, lama biliar ou microlitíase, que não foram adequadamente estudados, pelas razões já expostas ou mesmo por falta de recursos diagnósticos. A própria colangiografia endoscópica retrógrada, já tão antiga, não esteve disponível para permitir a melhor compreensão dos casos.

Os pacientes com pancreatite crônica agudizada foram incluídos porque apresentavam a mesma necrose, o mesmo quadro clínico e exigiram os mesmos cuidados dos pacientes das outras categorias ${ }^{4}$.

A indicação indiscutível foi a necrose infectada e raros casos de grandes massas necróticas, com infiltração de retroperitônio e que mesmo estéreis, comprometiam o estado geral dos pacientes ${ }^{2,5,6}$. O problema é distingüir entre necrose estéril e a infectada e isto é de grande importância clínica, porque a necrose estéril não exige tratamento cirúrgico de urgência, enquanto a infectada resulta em óbito, se protelada a abordagem $^{2}$. Balthazar et $a l^{7,8}$ e London et $a l^{9}$, deram importante contribuição para a interpretação das imagens tomográficas na pancreatite aguda grave e favoreceram o diagnóstico diferencial entre as formas estéreis e infectadas. Os achados clínicos e laboratoriais costumam ser semelhantes nas duas situações e por isto, para o diagnóstico pré-operatório da infecção, também tem sido preconizada a cultura do material, obtido por punção transcutânea.

Nesta série de operações, sempre houve preferência pelas incisões transversas, supraumbilicais, porque permitem uma abordagem mais específica do 
andar supramesocólico. A tentativa de usar grandes incisões e abdome aberto não apresentou vantagens, pois a mortalidade não foi menor nestes casos. Contudo, deve-se observar, que esta técnica foi utilizada somente nos casos mais graves. $\mathrm{Na}$ maioria das vezes foram utilizadas incisões com dez a quinze centímetros, que permitia o desbridamento do material necrótico, sem contaminar o andar inframesocólico. Esta incisões permaneciam abertas e tamponadas com compressas maiores ou menores, de acordo com o tamanho da incisão, conforme preconizam trabalhos de literatura ${ }^{10,11}$. Nos pacientes com abscessos retroperitoneais nos flancos, deu-se preferência às drenagens por abordagem extraperitoneal. Utilizava-se incisão transversa no flanco, cuja metade coincidia com a borda externa do músculo reto e se fazia o descolamento por trás do peritônio. Nesta técnica pode ocorrer perfuração do peritônio, pois nem sempre há plano de clivagem, mas isto não resultou em peritonite difusa. Até mesmo a drenagem retroperitoneal laparoscópica tem sido proposta, ainda que não utilizada na presente série ${ }^{12}$.

Pacientes operados por pancreatite aguda grave, necessitam freqüentemente de reoperações, para desbridamento de restos necróticos ou para tratar complicações ${ }^{3,13-17}$. Nesta série, 25 pacientes se submeteram a uma ou mais reoperações, mas este fato não representou aumento da mortalidade. Entende-se que as reoperações bem indicadas contribuam para reduzir os índices de morbimortalidade dos pacientes. Entre os reoperados, dez faleceram (40\%) e este índice não está acima do que se pode inferir da literatura. As operações foram realizadas em circunstâncias tais que a mortalidade seria total, se não indicadas.

A mortalidade hospitalar foi de $38,2 \%$ e assim é em alguns relatos da literatura, considerando-se que $70,6 \%$ dos pacientes foram operados por necrose infectada, quando o quadro se reveste de maior gravidade $^{18-20}$.

Em síntese, ocorreu um alto índice de operações por diagnóstico incerto, o que poderia ter sido evitado, atualmente, em vista dos avanços e sobretudo da disponibilidade dos recursos de diagnóstico por imagem. Esta indicação deve se restringir aos casos onde não seja possível o diagnóstico diferencial com certas causas de abdome agudo, como, por exemplo, o infarto êntero-mesentérico e alguns casos de colangite. Também as indicações para tratar precocemente a litíase biliar devem ser evitadas ou reduzidas a situações específicas, ainda com preferência para a abordagem endoscópica da papila. As reoperações são freqüentemente indicadas nesses pacientes e a infecção é a principal causa de morte.

\begin{abstract}
Background: To evaluate the results of surgical treatment of acute necrotizing pancreatitis, as well as its necrotizing alcoholic form. Methods: Patients were analyzed according to a prospective protocol. Surgery was performed on 68 patients due to necrotizing acute pancreatitis and acutized chronic pancreatitis from years 1982 to 2000. Patients were grouped into three categories: 1) Biliary acute pancreatitis; 2) Acute pancreatitis of unknown etiology; 3) Necrotizing alcoholic pancreatitis. Results: Patients had the following clinical indications for laparotomy: 1) Uncertain diagnosis (32.3\%); 2) Infected necrosis (60.3\%); 3) Necrosectomy (7.4\%). The surgical findings were: Infected necrosis (70.6\%); biliary complicatios (20.6\%) and large necrosis $1(7.4 \%)$. Global hospital mortality was $38.2 \%$. Individual categories showed a mortality of $33.3 \%$ (biliary acute pancreatitis), $45.0 \%$ (undetermined acute pancreatitis) and $37.0 \%$ (necrotizing alcoholic pancreatitis). Reoperations were indicated once or more times in 25 patients, and among these, the mortality was 40\%. Abscesses were the main cause of death (52.0\%), and all the other complications that resulted in death invariably evolved to infection. Conclusions: The indications of early surgical treatment for gallbladder stones, as well as when the diagnosis is not clear, should be reduced to very specific circumstances. Reoperations are common in these patients and infection was the main immediate cause of death.
\end{abstract}

Key Words: Pancreatitis, acute necrotizing; Surgery; Outcome assessment. 


\section{REFERÊNCIAS}

1. Read RC - "Management of primary acute pancreatitis". In Najarian JS, Delaney JP (eds) - Hepatic, biliary and pancreatic surgery. Chicago, Years Book, 1980, pp 265281.

2. Bradley EL - A clinically based classification system for acute pancreatitis. Summary of the International Symposium on Acute Pancreatitis, Atlanta, Ga, September 11 through 13, 1992. Arch Surg, 1993,128(5): $586-590$

3. Sarles H- "Alcoholic pancreatitis". In Burns GP, Bank $\mathrm{S}$ - Disorders of the pancreas. Current issues in diagnosis and management. New York, McGraw-Hill, 1992, pp. 273-282.

4. Takeda K, Matsuno S, Sunamura M, et al. - Surgical aspects and management of acute necrotizing pancreatitis: recent results of a cooperative national survey in Japan. Pancreas, 1998, 16(3):316-322.

5. Nakazaki H, Tajima T, Fujji K, et al. - A surgical treatment of infected pancreatic necrosis: retroperitoneal laparotomy. Dig Surg, 1999, 16(6):506-511.

6. De Waele JJ, Hesse UJ, Pattyn P, et al. - Postoperative lavage and on demand surgical intervention in the treatment of acute necrotizing pancreatitis. Acta Chir Belg, 2000, 100(1):16-20.

7. Balthazar EJ, Robinson DL, Megibow AJ, et al. - Acute pancreatitis: value of CT in establishing prognosis. Radiology, 1990, 174(2):331-336.

8. Balthazar EJ, Freeny PC, van Sonnenberg E - Imaging and intervention in acute pancreatitis. Radiology, 1994, 193(2):297-306.

9. London NJ, Neoptolemos JP, Lavelle J, et al. - Serial computed tomography scanning in acute pancreatitis: a prospective study. Gut, 1989, 30(3):397-403.

10. Bosscha K, Hulstaert PF, Hennipman A, et al. - Fulminant acute pancreatitis and infected necrosis: results of open management of the abdomen and "planned" reoperations. J Am Coll Surg, 1998, 187(3):355-262.

11. Farkas G, Marton J, Mandi Y, et al - Surgical strategy and management of infected pancreatic necrosis. $\mathrm{Br} \mathrm{J}$ Surg, 1996, 83(7):930-933.
12. Kasperk R, Riesener KP, Schumpelick V - Surgical therapy of severe acute pancreatitis: a flexible approach gives excellent results. Hepatogastroenterology, 1999, 46(25):467-471.

13. Piotrowski Z, Tolwinski W - Surgical treatment of severe necrotizing pancreatitis by the method of "open abdomen"-early and long-term results. Przegl Lek, 2000, 57 (Suppl 5):45-49.

14. Hut'an M, Salapa M - Methods of surgical treatment of necrotizing pancreatitis. Rozhl Chir, 2000, 79(10):484487.

15. Fernandez-del Castiho C, Warshaw AL - Parenchymal necrosis: infection and other indications for debridement and drainage. Chirurg, 2000, 71(3):269273.

16. Branum G, Galloway J, Hirchowitz W, et al. - Pancreatic necrosis: results of necrosectomy, packing, and ultimate closure over drains. Ann Surg, 1998, 227(6):870-877.

17. Gerzof SG, Banks PA, Robbins AH, et al. - Early diagnosis of pancreatic infection by Computed Tomography-guided aspiration. Gastroenterology, 1987,93(6):1315-1320.

18. Rasslan S, Kowes I, Silva RA, et al. - Severe acute pancreatitis, laparotomies, and planned reoperation. Rev Paul Med, 1990, 108(4):169-173.

19. Schmid SW, Uhl W, Friess $\mathrm{H}$, et al. - The role of infection in acute pancreatitis. Gut, 1999, 45(2):311-316.

20. Estakhri ME, Frey C - "Surgical treatment of pancreatic necrosis, abscesses, and cysts". In Burns GP, Bank S - Disorders of the pancreas. Current issues in diagnosis and management. New York, McGrawHill,1992, pp.186-204.

Endereço para correspondência:

Olívio Louro Costa

Rua Horácio Andrade de Carvalho, 210

Ilha do Boi

29052-620 - Vitória - Espírito Santo

E-mail: oliviolouro@terra.com.br 